\title{
A reabilitação psicossocial: processo de reconstrução da subjetividade do usuário de drogas
}

\author{
Psychosocial rehabilitation: process of reconstructing drug users' subjectivities \\ Rehabilitación psicosocial: proceso de reconstrucción de la subjetividad del usuario de drogas
}

\author{
Gustavo Costa de Oliveira ${ }^{\text {; }}$ Cíntia Nasili ; Annie Jeanninne Bisso Lacchinilit; \\ Marcio Wagner Camattal ; Carolina Maltz ${ }^{V}$; Jacó Fernando Schneider ${ }^{V I}$
}

\begin{abstract}
RESUMO: O estudo teve como objetivo compreender a reabilitação psicossocial como um processo de reconstrução da subjetividade do usuário de drogas na perspectiva da sociologia fenomenológica de Alfred Schutz. Trata-se de pesquisa qualitativa e fenomenológica, realizada de setembro de 2013 a fevereiro de 2014, com 12 trabalhadores de saúde de um Centro de Atenção Psicossocial álcool e drogas (CAPSad), no município de Porto Alegre, Rio Grande do Sul, Brasil. A coleta das informações ocorreu por entrevista fenomenológica e a análise, pela interpretação compreensiva. Identificou-se a importância do CAPSad na consolidação da reabilitação psicossocial como processo de reconstrução da subjetividade do usuário de drogas. O estudo evidenciou que se deve explorar o mundo da vida cotidiana do usuário de drogas e visualizar os contextos, através de sua visão, podendo-se compreender este indivíduo em sua singularidade e individualidade e, com isso, instituir ações em saúde mental que integrem, potencializem e tornem possível a reabilitação psicossocial.
\end{abstract}

Palavras-Chave: Enfermagem; saúde mental; psicossocial; filosofia.

ABSTRACT: The study aimed to understand psychosocial rehabilitation the reconstruction of drug users' subjectivities in a process informed by the phenomenological sociology of Alfred Schutz. This qualitative, phenomenological study of 12 health workers at a psychosocial care center (CAPSad), focused on alcohol and drug rehabilitation in Porto Alegre, Rio Grande do Sul State, Brazil. Data was collected by phenomenological interviews and analyzed using 'comprehending interpretation'. The CAPSad was found to be important to establishing psychosocial rehabilitation as a process to reconstruct drug users' subjectivities. The study showed that drug users' everyday worlds should be explored and contexts visualized as through their eyes in order to understand these individuals in their singularity and individuality and thus introduce mental health actions to integrate, potentiate and permit psychosocial rehabilitation.

Keywords: Nursing; mental health; psychosocial; philosophy.

RESUMEN: El estudio tuvo como objetivo comprender la rehabilitación psicosocial como un proceso de reconstrucción de la subjetividad del usuario de drogas en la perspectiva de la sociología fenomenológica de Alfred Schutz. Se trata de una investigación cualitativa y fenomenológica, realizada de septiembre 2013 a febrero 2014, junto a 12 trabajadores de salud de un Centro de Atención Psicosocial alcohol y drogas (CAPSad) en la ciudad de Porto Alegre, Rio Grande do Sul, Brasil. La recolección de datos fue puesta en marcha por medio de entrevista fenomenológica y análisis por interpretación comprensiva. Se ha identificado la importancia del CAPSad en la consolidación de la rehabilitación psicosocial como un proceso de reconstrucción de la subjetividad del usuario de drogas. El estudio mostró que se debe explorar el mundo de la vida cotidiana de los usuarios de drogas y ver los contextos a través de sus ojos, ser capaz de entender a esta persona en su singularidad e individualidad y, de este modo, establecer acciones en salud mental que integren, potencien y hagan posible la rehabilitación psicosocial.

Palabras Clave: Enfermería; salud mental; psicosocial; filosofía.

\section{INTRODUÇÃO}

A atenção em saúde mental, incluindo-se a atenção aos usuários de substâncias psicoativas, vem sendo redirecionada, nos últimos anos, para um modelo que privilegie o cenário da comunidade. Essa perspectiva surge com o movimento da reforma psiquiátrica, em que se preconiza uma atenção em saúde mental inserida em uma rede de serviços comunitários. Os Centros de Atenção Psicossocial (CAPS) possuem papel estra-

\footnotetext{
IMestre em Enfermagem. Doutorando em Enfermagem pelo Programa de Pós-Graduação em Enfermagem da Universidade Federal do Rio Grande do Sul. Porto Alegre, Rio Grande do Sul, Brasil. E-mail: gustavoenfufrgs@gmail.com.

"IDoutora em Enfermagem. Professora Adjunta do Curso de Enfermagem da Universidade Federal de Ciências da Saúde de Porto Alegre. Rio Grande do Sul, Brasil. E-mail: cintianasi@yahoo.com.br.

IIIDoutora em Enfermagem. Professora Adjunta do Curso de Enfermagem da Universidade Federal de Ciências da Saúde de Porto Alegre. Rio Grande do Sul, Brasil. E-mail: anniejbl@hotmail.com.

${ }^{\text {IV }}$ Doutor em Enfermagem. Professor Adjunto da Escola de Enfermagem da Universidade Federal do Rio Grande do Sul. Porto Alegre, Rio Grande do Sul, Brasil. E-mail:mcamatta@gmail.com.

vEnfermeira. Graduada pela Enfermagem da Universidade Federal de Ciências da Saúde de Porto Alegre. Rio Grande do Sul, Brasil. E-mail: carol.maltz@hotmail.com. VIDoutor em Enfermagem. Professor Titular da Escola de Enfermagem e do Programa de Pós-Graduação em Enfermagem da Universidade Federal do Rio Grande do Sul. Porto Alegre, Rio Grande do Sul, Brasil.E-mail: jaco_schneider@uol.com.br.
} 
tégico para a promoção de uma saúde mental voltada para o social, em que o tratamento não isola o usuário da sociedade, mas sim, busca atuar em interligação com os demais recursos da rede de saúde, visando à reabilitação psicossocial deste usuário ${ }^{1-3}$.

A reabilitação psicossocial configura-se como um processo que facilita a oportunidade para pessoas em sofrimento psíquico alcançarem um funcionamento com maior autonomia na comunidade. Em virtude de a reabilitação psicossocial apontar para a redução do estigma e preconceito e visar à maximização da saúde, seus proponentes estão empenhados na autoajuda, organização e suporte familiar, e promoção de esforços para fortalecer serviços e desenvolver redes e sistemas existentes ${ }^{4}$.

A reabilitação psicossocial da pessoa em sofrimento psíquico pode se caracterizar como um processo de reconstrução da subjetividade desta pessoa, considerando o seu habitat, rede social e trabalho, necessários para que consiga se (re)adaptar ao mundo 5 .

Na lógica da instituição da reabilitação psicossocial na terapêutica do usuário de drogas, as ações em saúde mental desenvolvidas nos CAPS álcool e drogas (CAP$\mathrm{Sad}$ ) visam fortalecer este sujeito, uma vez o processo terapêutico deve dispor ferramentas para (re)organização individual e (re)inserção na família e na sociedade.

Este estudo justifica-se pela importância de se compreender a reabilitação psicossocial como um processo de reconstrução da subjetividade dos usuários de drogas, dando-se a eles maior visibilidade. Nesta pesquisa, por meio da utilização do referencial teórico-metodológico da sociologia fenomenológica de Alfred Schutz, buscou-se dar voz aos trabalhadores de um CAPSad quanto às suas compreensões sobre o processo de reabilitação psicossocial dos usuários. De acordo com este referencial fenomenológico, consegue-se dar voz aos sujeitos, mergulhando-se em suas subjetividades e nas relações sociais, com intuito de desvelar o fenômeno a partir das vivências dos indivíduos ${ }^{6,7}$.

$\mathrm{O}$ estudo teve como objetivo compreender as intenções de trabalhadores quanto à reabilitação psicossocial no tratamento de usuários de um CAPSad.

\section{REVISÃo DE LITERATURA}

No Brasil, a assistência às pessoas em sofrimento psíquico e suas famílias era pautada no modelo hospitalocêntrico, centrado em atendimento na instituição asilar, com tratamento controlador e repressor, também executado pela equipe de enfermagem ${ }^{8}$. Na década de 1970, inicia-se um processo de mudança nesse modelo, denominado de reforma psiquiátrica brasileira.

A reforma psiquiátrica configura-se como o processo histórico de formulação crítica e prática, que tem como objetivos e estratégias o questionamento e a elaboração de propostas de transformação do modelo clássico e do paradigma da psiquiatria.

Os princípios, objetivos e pressupostos da reforma psiquiátrica orientam as ações na rede de saúde 4 . Pode-se pensar na reabilitação psicossocial da pessoa em sofrimento psíquico como uma proposta de introdução desse sujeito nas redes da comunidade, o que facilitaria sua inserção em seu meio social, implicando a possibilidade dele reconstruir os sentidos de sua vida cotidiana9.

A reabilitação psicossocial dos usuários de álcool e outras drogas pode ser propiciada nos serviços de saúde por meio de oficinas terapêuticas, em atividades externas e/ou parcerias estabelecidas pelo serviço, produzindo formas mais sociabilizantes de tratamento e possibilitando a circulação dos usuários pela cidade. Identifica-se que a reabilitação psicossocial dos usuários de substâncias psicoativas é um processo ainda complexo, que envolve várias dimensões, desde o nível micro (individual/familiar/institucional) até o macro (comunidade, sociedade e políticas de saúde mental). Para que esta reabilitação realmente se efetive, os serviços devem estar comprometidos com a transformação das condições de vida desses sujeitos ${ }^{10}$.

O conceito de reabilitação psicossocial refere-se não apenas ao apoio ou suporte da rede social do usuário, mas implica uma conceituação mais abrangente, relacionada à transformação do sujeito de sua condição de doente para o seu papel de cidadão. No contexto da Reforma Psiquiátrica, a reabilitação psicossocial deve procurar restituir a subjetividade do indivíduo, possibilitando a recuperação do seu poder de contratualidade, no sentido de desenvolver estratégias e ações intra-setoriais e intersetoriais efetivas, com o propósito de que estes possam assumir sua condição de sujeito social ${ }^{11}$.

No cenário atual, os serviços substitutivos surgem como serviços que buscam consolidar o modo de atenção psicossocial, operando com outra conotação teórica que não somente a da psiquiatria clínica. Seus pressupostos destacam o atendimento aos sujeitos em sofrimento psíquico, assim como a reabilitação psicossocial, visando promover o exercício da cidadania e a interação social ${ }^{12}$.

\section{Referencial Teórico-Metodológico}

Trata-se de uma pesquisa qualitativa, de abordagem fenomenológica, com o referencial teórico-metodológico da sociologia fenomenológica de Alfred Schutz.

O cenário deste estudo foi o CAPSad III, ligado ao Grupo Hospitalar Conceição (GHC), localizado no município de Porto Alegre, Rio Grande do Sul, Brasil.

Os sujeitos do estudo são 12 trabalhadores do CAPSad, das diferentes categorias profissionais e dos três turnos de trabalho do serviço. Os critérios de inclusão dos trabalhadores da equipe foram: fazer parte do quadro funcional do serviço; ser maior de idade; estar atuando no serviço há, pelo menos, três meses. 
A coleta das informações foi realizada por meio de entrevista fenomenológica, no próprio CAPS, no período de setembro de 2013 a fevereiro de 2014. As entrevistas tiveram as seguintes questões norteadoras: Qual a sua concepção sobre a atenção ao usuário de drogas; Conte para mim que ações você desenvolve no CAPS; e $\mathrm{O}$ que você tem em vista com essas ações?

As informações obtidas foram submetidas à análise fenomenológica, em consonância com o referencial de Alfred Schutz. Para desvelar as vivências expressas nos depoimentos dos trabalhadores acerca do significado do atendimento em saúde mental, as falas foram analisadas conforme indicação de pesquisadores da fenomenologia social ${ }^{13,14}$ : realizar leituras e releituras dos conteúdos das falas objetivando obter a essência do significado das ações dos trabalhadores; identificar trechos das falas que apresentem o significado da ação de oferecer atendimento no CAPSad; agrupar os aspectos em comum das unidades de significado, isto é, as convergências que possibilitam o emergir das categorias concretas acerca das ações estabelecidas entre trabalhadores que atuam no CAPS.

Após a coleta dos dados, buscou-se a convergência das informações presentes nas falas dos entrevistados, submetendo-as à análise fenomenológica.

A fim de garantir o anonimato dos sujeitos entrevistados na pesquisa, os trabalhadores da equipe foram identificados na redação pela sigla $E$, acompanhada do número correspondente à ordem das entrevistas (de E1 a E12).

Para o desenvolvimento da pesquisa, foram seguidos os aspectos éticos necessários ao desenvolvimento de pesquisas com seres humanos, conforme a Resolução $n^{\circ}$ 466/2012 do Conselho Nacional de Saúde do Ministério da Saúde ${ }^{15}$. Assim, foi distribuído, anteriormente, o Termo de Consentimento Livre e Esclarecido para os participantes da pesquisa, em duas vias, ficando uma em poder do participante e outra do pesquisador.

$\mathrm{O}$ projeto de pesquisa foi encaminhado à Plataforma Brasil e recebeu aprovação do Comitê de Ética em Pesquisa da Universidade Federal de Ciências da Saúde de Porto Alegre, conforme Parecer n ${ }^{\circ} 296.058$, e do Comitê de Ética em Pesquisa do GHC, conforme Parecer $n^{\circ} 371.804$.

\section{Resultados e Discussão}

A partir da análise dos depoimentos à luz do referencial da fenomenologia social de Alfred Schutz, apresenta-se a categoria concreta: Reabilitação psicossocial no CAPSad.

\section{Reabilitação psicossocial no CAPSad}

Compreender o ser humano é uma tarefa complexa, uma vez que há questões subjetivas, biológicas, psicológicas e sociais ${ }^{7}$. Além disso, compreender con- figura-se como a forma experiencial do conhecimento que o senso comum tem das coisas ${ }^{6}$. Nessa perspectiva, a compreensão sociológica é o resultado da interpretação subjetiva do pesquisador fenomenológico a respeito do fenômeno de conduta humana que este estuda?

\section{Motivos da ação}

Entretanto, para este pesquisador compreender a ação do outro é necessário conhecer os motivos para de seu ato, reconhecendo que uma ação social envolve atitudes e agir de pessoas, orientadas para o ato do outro?

Dessa maneira, os motivos que conduzem à ação do ator social podem ter um significado subjetivo (motivos para) e outro objetivo (motivos porque) ${ }^{7}$, sendo que os motivos para delinearam, nesta pesquisa, a interpretação das ações sociais dos sujeitos no mundo da vida cotidiana.

Inicialmente, observou-se nas falas dos trabalhadores de saúde do CAPSad, que estes:

O objetivo tu sempre vê que é melhora do paciente. (E6)

Poder colaborar com que ele melhore a vida dele. (E10)

As falas evidenciam que o processo terapêutico no CAPSad, ao olhar dos profissionais, se constitui com o propósito de melhorar a situação de saúde do usuário. As práticas em saúde mental buscam contribuir com a melhora da vida deste usuário, o que pode significar intervenções em saúde que perpassam os muros do serviço de saúde.

Outro elemento presente na narrativa dos trabalhadores de saúde é a expectativa pela melhora dos usuários. Nas falas a seguir, os profissionais relatam o seu desejo de que o tratamento proporcione a melhora do estado de saúde destes usuários:

A gente espera que o paciente fique bom [...] a gente fica contente de saber que pacientes nossos que começaram tratamento, agora estão bem. (E5)

Eu espero que o usuário fique bem assim, independente da escolha que ele faça, se vai ser de um uso, ou se vai ser de uma abstinência, da interrupção desse uso. (E11)

Continuo esperando que eles melhorem.

$(E 12)$

Com base nas falas, nota-se a conexão entre as ações em saúde mental desenvolvidas no CAPSad e as expectativas dos trabalhadores de saúde quanto ao prognóstico do usuário. Desse modo, o serviço de saúde pode propiciar um cuidado em saúde voltado ao usuário, valorizando-se as subjetividades, caracterizando este usuário como um sujeito social, dotado de valores e crenças, os quais podem influenciar a terapêutica.

\section{Autonomia dos clientes}

Esse cuidado em saúde poderia proporcionar a compreensão do ser humano situado no seu mundo de vida, partindo da realidade concreta, isto é, das suas experiências vivenciadas. $\bigcirc$ mundo da vida é o mundo 
social, que é compartilhado, vivenciado e interpretado por todos os atores, sendo que é nele que coexistem semelhantes com quem se estabelecem diferentes relações sociais, podendo-se produzir sujeitos-cidadãos que possam ser responsáveis em gerir suas vidas?

Alguns trabalhadores de saúde expressaram a necessidade de se construir ações em saúde que promovam autonomia aos usuários, como se visualiza nas falas a seguir:

Trabalhar autonomia para que ele consiga reorganizar a sua vida sem estar dependendo dos serviços e nem do uso abusivo de substâncias. (E3)

Oferecer um cuidado para esse usuário, mas que esse cuidado não seja apenas dentro de um serviço de saúde, mas que esse cuidado também vá para fora. (E7)

Fortalecer essa autonomia deles, fortalecer eles enquanto pessoas [...] Pensar juntos o que a gente vai fazer, o que ele vai fazer, e [...] tentar fazer que ele construa a saída pra sair dessa. (E10)

A autonomia dos usuários, estabelecida ao longo do processo terapêutico, pode promover a (re) construção de sua vida, fortalecendo-os no mundo da vida cotidiana. A partir dos depoimentos, percebe-se que a autonomia está atrelada a ações em saúde que auxiliem os usuários nas próprias escolhas no cotidiano e na organização de suas vidas.

Nessa direção, para a construção de uma abordagem psicossocial voltada para a autonomia dos usuários, é necessária a criação de instrumentos que potencializem o sujeito para a produção de projetos de vida, no sentido de independência terapêutica. Assim, as práticas em saúde mental devem ser articuladas ao contexto social do indivíduo, em que habilidades podem ser potencializadas e, com isso, também contribuam para o reconhecimento de autonomia do ator social ${ }^{16}$.

Outros trabalhadores mencionaram, no campo das intenções, a construção da autonomia dos usuários a partir de ações em saúde, com o propósito de fortalecer habilidades e potencialidades, bem como auxiliar no processo decisório destes indivíduos, conforme as falas que seguem:

[...] ver mais questões de habilidades e potencialidades. (E11)

[...] tento ocupar esses espaços fazendo com que ele desenvolva [...] habilidades cognitivas, [...] para que depois, quando ele retorne para sua casa ou para a sociedade, ele possa ocupar alguma coisa. (E1)

Tentar organizar eles para que eles possam viver lá fora, [...] e tentar mostrar para eles que eles podem, sim, fazer escolhas boas, escolhas que vão levar eles a ter uma vida diferente, melhor do que eles vinham tendo. (E2)

\section{Reinserção social}

Ao analisar as falas, nota-se que os trabalhadores buscam, por meio de ações em saúde, apurar habilidades e potencialidades dos usuários, com intuito de desenvolver formas que sensibilizem a sociedade para a compreensão destes usuários, e se responsabilize, também, pelo processo de inserção social.

A autonomia parece identificar-se com as relações sociais, podendo-se inserir socialmente indivíduos segregados, através da minimização dos efeitos do sofrimento psíquico e do incentivo ao usuário para o enfrentamento de seus problemas ${ }^{17}$. Entretanto, essa autonomia tem por base suas condições sociais, econômicas, culturais e o resgate de sua cidadania, para a própria reinserção na sociedade ${ }^{18}$.

Ao se considerar o contexto em que este usuário está inserido, atentando para o mundo de vida cotidiana de cada indivíduo, pode-se favorecer a transcendência de valores, a intersubjetividade e a interação social ao longo do processo ${ }^{6,7}$. Dessa forma, a reinserção social do usuário torna-se essencial para que este possa compartilhar o mundo da vida cotidiana com seus semelhantes, reconstruindo vínculos e refletindo sobre o cotidiano para organizar suas escolhas, como pode ser observado nos registros a seguir:

Reinserir ele na sociedade, [...] oferecer esse espaço para que ele possa refletir sobre tudo que ele fez, [...] e o que ele quer da vida dele. (E1)

Aqui seria um espaço para que as pessoas possam se reciclar, [...] pode reconstruir vínculos [...], tu pode voltar a viver em sociedade. [...] aqui é uma preparação para que a nossa clientela retorne à sociedade. (E2)

O objetivo principal é [...] que ele consiga reintegrar para a sociedade. (E6)

Nota-se que o trabalho em saúde no CAPSad propõe a construção de espaços para a inclusão social dos usuários, nos quais incita-se a reflexão, a educação em saúde e a redução do uso de substâncias psicoativas. Com isso, na visão dos trabalhadores, pode-se preparar estes usuários para a reintegração à sociedade.

mundo social configura-se como um ambiente intersubjetivo, que abrange todas as esferas das vivências e experiências cotidianas, por meio das quais os sujeitos, por suas intenções, relacionam-se com seus semelhantes, projetam ações e concebem planos. É no mundo da vida que se organiza a experiência a partir do presente e se direciona ao passado e ao futuro, e que há o compartilhamento de significados nas interações sociais?

Por essa razão, considera-se crucial a aproximação ao usuário do serviço de saúde, com base em um trabalho em saúde essencialmente interdisciplinar, viabilizando um processo terapêutico que atenda a todos os interesses ${ }^{19}$. Além disso, este trabalho em saúde poderá permitir que o usuário se readapte ao desenvolvimento de atividades cotidianas como trabalhar, estudar, passear, isto é, que o indivíduo consiga gerenciar sua vida, conforme se visualiza nas falas a seguir.

Que volte para sua rotina, que volte a trabalhar, volte com seus familiares. (E5) 
Ela arrumar um emprego, ter uma boa vida de relação com a família, com a esposa, com os filhos. (E12)

Eu espero que ele consiga [...] trabalhar, consiga gerenciar o seu dinheiro para poder dar condições para a família. (E6)

É importante compreender que a reinserção social, consubstanciada no processo terapêutico e nas relações sociais entre todos os atores, pode fortalecer o lastro social das ações nos serviços de saúde. Dentro dessa compreensão, a inserção social torna-se o lócus para a reflexão do mundo da vida cotidiana dos usuários, sendo um dos dispositivos para realização de práticas que possam promover a transformação da realidade social.

Os dispositivos do cuidado em saúde mental se transversalizam no cotidiano do CAPS, principalmente nas interações sociais construídas no decorrer do processo terapêutico ${ }^{18}$. Nesse cenário, ressalva-se a reabilitação psicossocial, a qual requisita dos atores envolvidos uma maior capacidade de articulação com as possibilidades de atenção em saúde mental do serviço de saúde ${ }^{20}$ e com as próprias demandas que constituem o contexto subjetivo de cada usuário, familiar e trabalhador.

Assim, salienta-se que as ações em saúde mental desenvolvidas no CAPSad propõem a construção de uma terapêutica voltada para a melhora do estado de saúde do usuário, sua autonomia e reinserção social. Com isso, o trabalho em saúde pode auxiliar na constituição da reabilitação psicossocial dos usuários, em que estes possam participar ativamente do cuidado em saúde mental, e sejam protagonistas do seu mundo de vida cotidiana.

\section{ConClusão}

Nesta pesquisa, observou-se, a partir da análise das falas dos trabalhadores de um CAPSad, a instituição de ações em saúde mental nesse espaço, com o intuito de reabilitação psicossocial do usuário no processo terapêutico. Percebeu-se que, neste cuidado, torna-se primordial o relacionamento social entre o profissional de saúde e o usuário, o que pode permitir o fortalecimento do vínculo e o entendimento do contexto social do usuário de drogas, trabalhando-se a reabilitação psicossocial como um processo de reconstrução da subjetividade deste sujeito.

Os resultados deste estudo demonstraram que o processo terapêutico se apresenta com propósito de melhorar a situação de saúde do usuário; de estimular a autonomia dos usuários para propiciar melhores escolhas e organização no cotidiano; de preparar os usuários de drogas para o convívio em sociedade por meio da apuração de habilidades; e conectado às expectativas dos profissionais. No entanto, deve-se refletir sobre estas características da terapêutica no CAPSad, valorizando tais propostas de assistência ao usuário de drogas e, principalmente, avaliando se há dificuldades na sua estruturação e execução.

Soma-se a esta perspectiva, a busca, por parte dos profissionais de saúde do CAPSad, da organização do cuidado em saúde mental com vistas à reabilitação psicossocial, a partir da aproximação ao contexto social de cada usuário de drogas. Para tanto, é essencial explorar o seu mundo da vida cotidiana e visualizar os contextos, podendo-se compreender este indivíduo em sua singularidade e individualidade e, com isso, instituir ações em saúde mental que integrem, potencializem e tornem possível o processo de reconstrução da subjetividade do usuário de droga, isto é, a reabilitação psicossocial.

\section{REFERÊNCIAS}

1.Dutra VFD, Rocha RM . O processo de desinstitucionalização psiquiátrica: subsídios para o cuidado integral. Rev enferm UERJ. 2011;19:386-91.

2.Schrank G, Olschowsky A. O centro de Atenção Psicossocial e as estratégias para inserção da família. Rev esc enferm USP. 2008;42:127-134.

3.Amarante P. Loucos pela vida: a trajetória da reforma psiquiátrica no Brasil. 2a.ed. Rio de Janeiro: Editora Fiocruz; 2009.

4.Hirdes A. Reabilitação psicossocial: dimensões teóricopráticas do processo. Erechim(RS): EdiFAPES; 2001.

5.Pitta AMF. Reabilitação psicossocial no Brasil. São Paulo: Hucitec; 2001.

6.Schutz A. Fenomenologia e relações sociais: textos escolhidos de Alfred Schutz. Compilação de Helmul Wagner. Rio de Janeiro: Zahar; 1979.

7.Schutz A. Sobre fenomenologia e relações sociais. Tradução de Raquel Weiss. Petrópolis(RJ): Editora Vozes; 2012.

8.Almeida Filho AJ, Moraes AEC, Peres MAA. Atuação do enfermeiro nos centros de atenção psicossocial: implicações históricas da enfermagem psiquiátrica. Rev RENE. 2009; 10(2):158-165.

9.Oliveira GC. Expectativas de familiares sobre uma unidade de internação psiquiátrica [dissertação]. Porto Alegre (RS): Universidade Federal do Rio Grande do Sul; 2014.

10.Pinho PH, Oliveira MAF, Vargas D, Almeida MM, Machado AL, Silva ALA et al . Reabilitação psicossocial dos usuários de álcool e outras drogas: a concepção de profissionais de saúde. Rev esc enferm USP 2009; 43:1261-6.

11.Pinho PH, Oliveira MA, Almeida MM. A reabilitação psicossocial na atenção aos transtornos associados ao consumo de álcool e outras drogas: uma estratégia possível? Rev Psiq Clin. 2008;35 (supl 1):82-8.

12.Nasi C, Schneider JF. O Centro de Atenção Psicossocial no cotidiano dos seus usuários. Rev esc enferm USP. 2011; 45:1157-63.

13.Lima CA, Tocantins FR. Necessidades de saúde do idoso: perspectivas para a enfermagem. Rev Bras Enferm. 2009; 62:367-73. 
14.Nasi C. As expectativas dos usuários e as intenções dos trabalhadores de um Centro de Atenção Psicossocial [tese de doutorado]. Porto Alegre (RS): Universidade Federal do Rio Grande do Sul; 2011.

15.Ministério da Saúde(Br). Conselho Nacional de Saúde. Resolução n ${ }^{\circ}$ 466, de 12 de dezembro de 2012: diretrizes e normas regulamentadoras de pesquisa envolvendo seres humanos. Brasília(DF): Ministério da Saúde; 2012.

16.Alves ES, Francisco AL. Ação psicológica em saúde mental: uma abordagem psicossocial. Psicol Cienc Prof. 2009; 29:768-79.

17.Fleury-Teixeira P, Vaz FAC, Campos FCC, Álvares J, Aguiar RAT, Oliveira VA. Autonomia como categoria central no conceito de promoção da saúde. Ciênc saúde coletiva. 2008; 13:2115-22.

18.Jorge MSB, Pinto DM, Quinderé PHD, Pinto AGA, Sousa FSP, Cavalcante CM. Promoção da saúde mental - tecnologias do cuidado: vínculo, acolhimento, co-responsabilização e autonomia. Ciênc saúde coletiva. 2011; 16:3051-3060.

19.Furtado JP, Nakamura E, Generoso C, Guerra A, Campos FB, Tugny A. Inserção social e habitação: um caminho para a avaliação da situação de moradia de portadores de transtorno mental grave no Brasil. Interface - Comunic, Saude, Educ. 2010; 14:389-400.

20.Loyola CMD, Pôrto KF, Rocha KS. Implicações do programa nacional de avaliação do sistema hospitalar psiquiátrico para a enfermagem psiquiátrica. Rev enferm UERJ. 2009; 17:9-13. 